\title{
Keeping tabs on fluorescent tags
}

\section{Fluorescence-based reagents are quickly evolving to keep pace with the demands of many research disciplines that have grown to depend on them. Caitlin Smith takes a look at recent developments.}

It took more than 30 years to clone GFP from jellyfish, but since this protein came on the stage in 1992, it has become a staple in cell biology laboratories around the world. Today, new fluorescent proteins are popping up every year. A year ago, a feature in Nature Methods ${ }^{1}$ highlighted the diverse fluorescent reagents available from a variety of companies, which develop their own fluorescent tools or commercialize the oeuvre of specialized academic labs. But developments are occurring so fast that impressive advances have reared their heads in the interim. Some of the interesting reagents that were not distributed commercially a year ago can now be ordered from manufacturers' catalogs, and new developments in academic labs have the promise to upstage current tools. Here is an update, focusing on fluorescent tags, photoswitchable fluorescent proteins and biosensors.

\section{Keeping track}

At their most basic function, fluorescent proteins are easy-to-detect tags for your favorite protein. For researchers, the commercialization of new fluorescent proteins means, first of all, a wider palette of colors and features is available for their experiments.

Among the new offerings on the market, the Organelle Lights reagents from Molecular Probes-which was recently acquired by Invitrogen -were introduced as genetically encoded organelle-specific labels. "Organelle Lights reagents are ideal tools for gaining detailed understanding of cellular organization and dynamic processes with high spatial and temporal resolution," says Magnus Persmark, labeling and detection project manager at Molecular Probes-Invitrogen. The reagents are fusions of fluorescent proteins with signal peptides that direct the

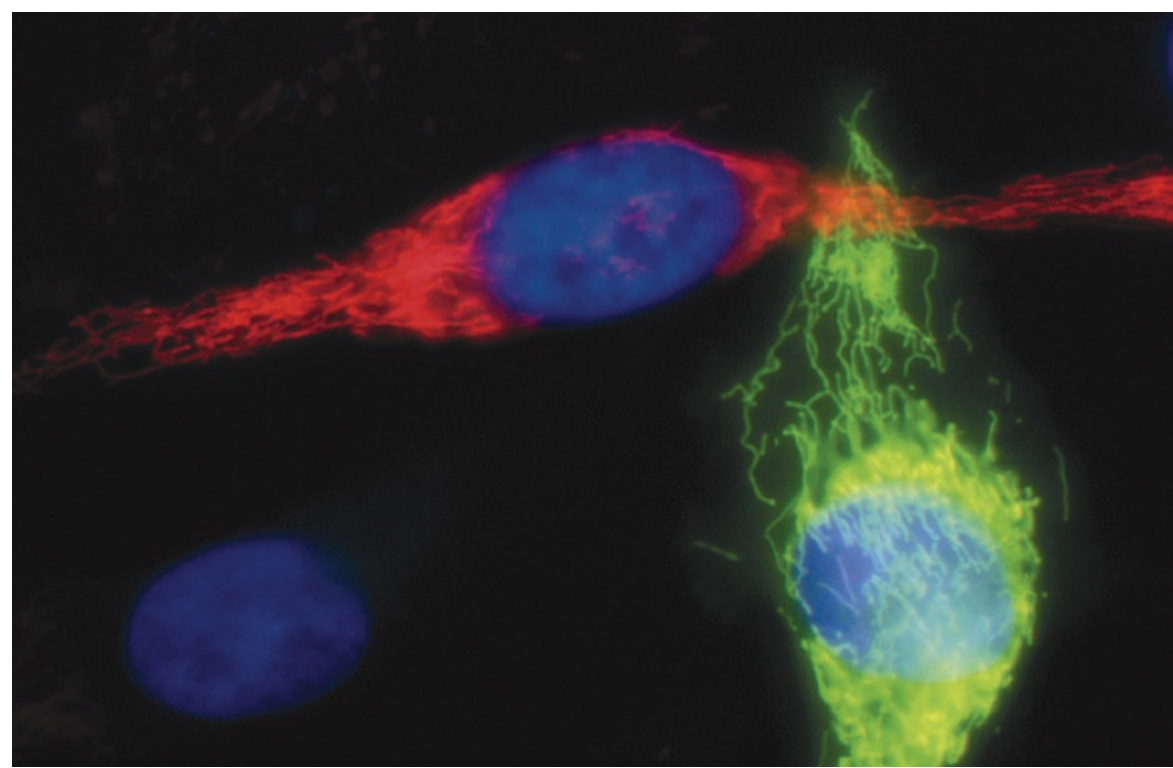

Organelle Lights Mito-GFP and Mito-OFP labeling of HeLa cells. (Courtesy of Invitrogen.)

fluorescent marker to specific intracellular compartments, and they are supplied with BacMam, a baculovirus-based gene delivery system. Four fluorescent protein colors-cyan, green, yellow and orangehave been engineered to reach a variety of cellular targets, including the nuclear and plasma membranes, endoplasmic reticulum, Golgi and peroxisomes.

Several other companies recently launched new fluorescent protein variants as well. For example, Evrogen sports the multicolor Turbo and Tag product lines. The company recommends the Turbo line, based on dimeric fluorescent protein labels, for cell and organelle labeling as well as tracking promoter activity, whereas the Tag line, based on monomeric labels, is recommended for creating fusion proteins. Also, Clontech has recently added to its portfolio of fluorescent proteins the 'fruit proteins' developed in the lab of Roger Tsien at the University of California
San Diego: mStrawberry, mPlum, mRaspberry, mBanana, mOrange and mCherry, along with specific antibodies to these fluorescent proteins to help with experimental validation. MBL International is offering CoralHue Keima-Red, which Suzan Oberle, MBL's director of marketing, describes as "a red fluorescent protein with the longest Stokes shift of any commercially available fluorescent protein." She adds that with its far-red emission at $620 \mathrm{~nm}$, it is especially useful as a tag in multicolor fluorescence experiments with labeled proteins or organelles. Yet another player, Stratagene, released new and improved versions of its Vitality fluorescent tag-for example, the hrGFPII, which is brighter than the previously released hrGFP.

Fluorescent proteins are also included in commercial-vector products intended for easy delivery and detection of a gene of interest. Stratagene now offers Vitality 
IRES biscistronic vectors for the coexpression of a protein of interest and a reporter from a single transcript; the reporters available are hrGFPII or their new orange fluorescent protein, cOFP. And recently, Clontech brought two retroviral bicistronic vectors to the market: the pRetroXIRES-DsRed-Express and pRetroX-IRESZsGreen 1.

Other fluorescent tags that are not based on fluorescent proteins have been developed in parallel (Box 1).

\section{Now you see them, now you do not}

Once you maneuver your fluorescent protein to a location of interest, what can it do? Once upon a time, these proteins simply fluoresced in response to a certain wavelength of light, but now some are capable of very clever tricks.

Photoswitchable fluorescent proteins are proving to be valuable tools for labeling and tracking specific parts of cells, such as individual organelles. Although there are many types of photoswitchable fluorescent proteins, all of them share the trait that light of a specific wavelength and intensity can change their spectral properties. Upon exposure to light with these specific attributes, photoac- tivatable fluorescent proteins undergo a reversible or irreversible change from a dark to bright fluorescent state, whereas photoconvertable fluorescent proteins undergo a generally irreversible change in color (that is, their emission wavelength changes). Photoactivable proteins can be switched from an 'off' (dark fluorescence) to an 'on' (bright fluorescence) state, upon exposure to certain light, and for some of these proteins the change is reversible. Photoconvertable proteins, in contrast, can change color as their emission wavelength is changed in response to specific light, but this change is usually irreversible.

Kindling red fluorescent protein (KFPRed) from Evrogen is a high-contrast photoactivatable red fluorescent protein used for observing direct movement of cells and organelles in vivo. The fluorescence is activated, or kindled, by a green laser that is less damaging to cells or tissues than the shorter-wavelength lasers that are required for the majority of photoswitchable fluorescent proteins. KFP-Red can be kindled reversibly or irreversibly depending on the intensity of the activating light; reversible kindling, which can be repeated indefinitely, is suitable for short-term tracking,

\section{BOX 1 PROMEGA'S HALOTAG SYSTEM PLUS FLUORESCENT PROBES}

In Promega's HaloTag system, the protein of interest is tagged with a derivative of a prokaryotic hydrolase rather than with a fluorescent protein. Under physiological conditions this hydrolase variant is capable of forming a covalent link with one of several specifically engineered synthetic ligands. A variety of ligands are available that contain fluorescent dyes or a biotin moiety to serve as detection label, or affinity handle.

The system has been available from Promega for a few years, but they have recently developed a new fluorescent ligand, HaloTag Alexa Fluor 488, which does not cross the cell membrane and thus labels the protein of interest only if and when it is expressed on the surface of the cell. "That way

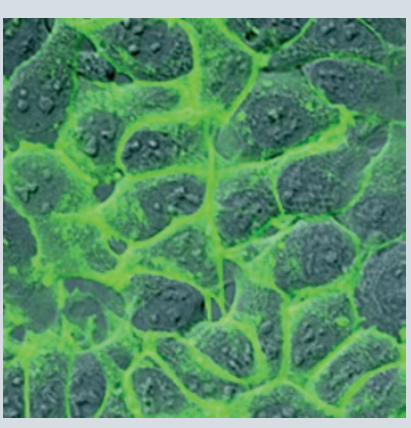

$\beta 1$-integrin labeled with the HaloTag protein and the new HaloTag Alexa Fluor 488 ligand. (Courtesy of Promega.) you can localize where the protein of interest is when it is on the surface," explains Bob Bulleit, director of research and development at Promega.

Using two fluorophores of different colors, it is also possible to label different pools of the same protein at different times. If labeling with Alexa Fluor 488, which is green, is followed by labeling with a second fluorophore that is red, the green and red labels will correspond to proteins processed from the Golgi or endoplasmic reticulum out to the cell membrane at different times.

whereas irreversible kindling is ideal for longer-term tracking. After reversible kindling, KFP-Red can either, with time, be allowed to relax to its initial nonfluorescent form or be quenched instantly with blue light. A reversibly kindled KFP-Red fluoresces at least 70 times brighter than the unkindled protein. After irreversible kindling KFP-Red gives stable red fluorescence that is at least 35 times brighter than that of the protein before kindling, and will continue to fluoresce for more than 72 hours in living cells and for at least a year in protein samples.

Evrogen's PS-CFP2 is an improved version of the photoconvertable fluorescent protein PS-CFP that matures faster and fluoresces more brightly both before and after photoswitching. In response to light activation, PS-CFP2 switches from cyan to green. According to Evrogen, the complete photoconversion results in a more than 2,000-fold increase in the greento-cyan fluorescence ratio. The high $\mathrm{pH}$ stability of PS-CFP2 makes targeting to acidic environments of endosomes and lysosomes possible. Evrogen also offers Dendra2, a photoconvertable fluorescent protein that switches from green to red fluorescence and offers even greater contrast photoconversion than PS-CFP2, with a more than 4,000-fold increase in the red-to-green fluorescence ratio after activation with blue light.

MBL International also offers two photoconvertable proteins, CoralHue Kaede and CoralHue Kikume (KikGR1), which both convert from green to red in response to photoactivation. The red fluorescence is comparable in intensity to the green fluorescence and is stable under usual aerobic conditions. KikGR1 is several-fold brighter and is more efficiently photoconverted than CoralHue Kaede.

\section{FRETting over sensors}

Just over ten years ago, the routine use of fluorescence resonance energy transfer (FRET) might have been unthinkable. In FRET, energy can be transferred from an excited fluorophore to a nonexcited one that is within several angstroms. Biosensors can be constructed so that events such as ligand binding, phosphorylation or cleavage cause a conformational change that brings together two FRET partners (fluorophores) that are attached to the sensor, resulting in a measurable change in the fluorescence ratio of donor and acceptor fluorophores. 
These usually small changes in the fluorescence signal can be difficult to detect, but devices that allow simultaneous imaging of two spectral channels, such as the Dual-View and Dual-Cam imaging systems from MAG Biosystems, simplify ratiometric imaging of potentially fast but small changes in FRET and can be easily attached to the camera port of a micro-

scope. FRET sensors have been constructed for assaying calcium signaling, receptor tyrosine kinases, intracellular kinases and histone methylation, just to name a few, not only in vitro but also in living cells in real time.

"In the long run, I believe that use of fluorescent-protein biosensors to study chemical changes within the cell in real time will lead to dramatic breakthroughs," says Jim Remington professor of physics in the Institute of Molecular Biology at the University of Oregon. "For example, one can imagine visualizing within a single cell, signal transduction events that take place in response to an external influence or visualizing localized chemical changes that take place during metabolic transitions." Many research groups, including Remington's, have produced fluorescent proteins that change color in response to changes in oxygen concentration, redox potential, $\mathrm{pH}$, calcium concentration or voltage gradients, for example.

A handful of FRET-based sensors have already made their way to the market. One of them is the Premo Cameleon calcium sensor, now available from Molecular Probes-Invitrogen. Based on a GFP sensor family developed by the Tsien and Miyawaki labs, the Premo Cameleon sensor undergoes a conformational change when its calmodulin moiety binds four calcium ions. This structural change brings the cyan and yellow fluorescent protein domains close enough together for FRET to occur.

Another FRET-based sensor is Evrogen's HyPer, which affords ratiometric measurement of intracellular hydrogen peroxide. It is the first fully genetically encoded fluorescent sensor capable of high specific detection of intracellular hydrogen perox-

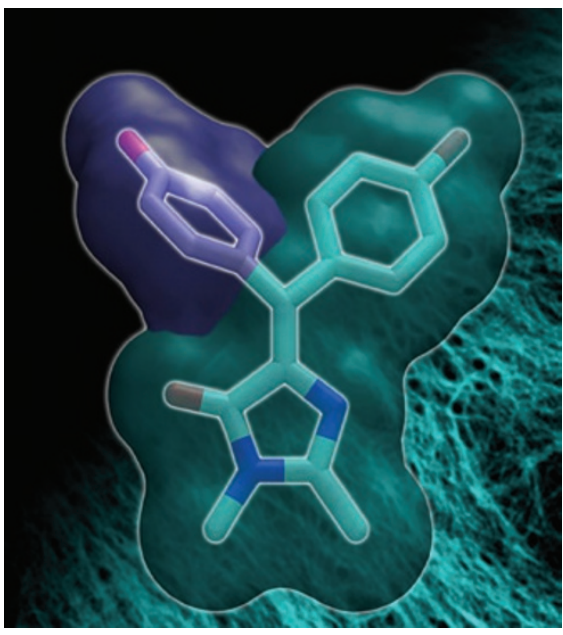

A photoswitchable fluorescent protein in the off and the on state. (Courtesy of Jim Remington.)

ide $\left(\mathrm{H}_{2} \mathrm{O}_{2}\right)$, one of the main reactive oxygen species generated by cells.

VisEn Medical has a proprietary fluorescence imaging probe platform that includes the MMPSense probe to detect matrix metalloprotease (MMP) activity 
in vivo. "This combines FRET-type activation, with optimized in vivo delivery and pharmacokinetic characteristics." says Kirtland Poss, president and CEO of VisEn Medical. VisEn Medical has also developed a near-infrared fluorescence probe for imaging the activity of cathep$\sin \mathrm{K}$, a cysteine protease that appears to be involved in the formation and destabi-

lization of atherosclerotic plaques.

"The beauty of many of these sensors is the lack of biological activity, which allows for long-term expression," says Moritz Bünemann, a professor of pharmacology and toxicology at the University of Würzburg in Germany, whose lab is using FRET-based sensors to investigate the mechanisms of drug action on $G$ proteincoupled receptors. Bünemann, however, sees challenges to be overcome for FRETbased sensors to make a big impact on drug development. One of the major challenges is the adaptation of high-throughput screening instruments to the needs of FRET. Says Bünemann, "The brightness of FRET probes also needs to be increased as well as the bleaching to be reduced in order to increase signal-to-noise [ratio] of FRET probes."

As more fluorescent proteins become available, better FRET probes will likely follow. Vladislav Verkhusha, associate professor in the department of anatomy and structural biology, at the Albert Einstein College of Medicine, notes the recent development of two bright and very photostable blue fluorescent proteins: Azurite ${ }^{2}$ and EBFP2 (ref. 3). "The trend will be making biosensors based on a FRET between these enhanced blue fluorescent proteins and already optimized/enhanced green fluorescent proteins such as EGFP and TagGFP," says Verkhusha. "This will allow using a second pair of fluorescent proteins such as orange fluorescent proteins (mOrange, $\mathrm{mKO}$ ) and red fluorescent proteins (mCherry, TagRFP) for the second FRET in the same mammalian cell. Thus, two independent processes or metabolites or interactions will be detected simultaneously."

Despite some limitations and difficulties of use, FRET-based reagents are also being used to monitor protein-protein interactions (Box 2).

\section{No FRET sensors}

FRET-based sensors are not the only fluorescent reagents designed to sense small molecules, events and cellular conditions. Verkhusha's lab is now developing socalled 'relocalization fluorescent biosensors', which move from one cell location to

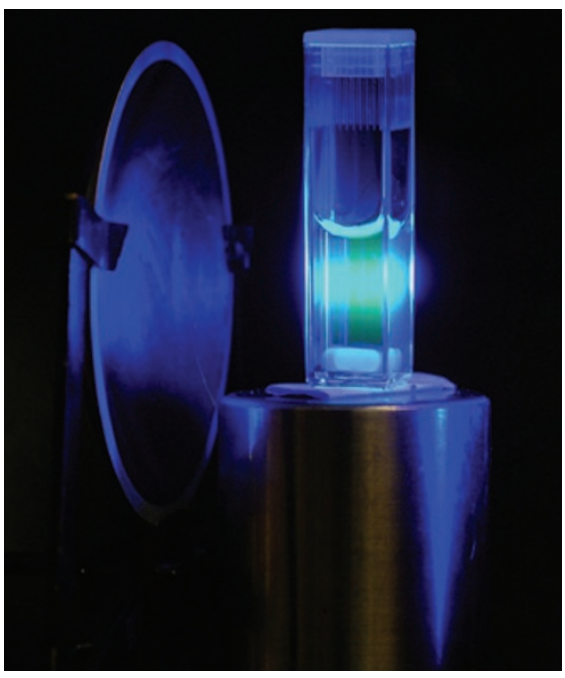

A redox-sensitive GFP. (Courtesy of Jim Remington.)

the other, usually between cytoplasm and membrane, upon a change in local conditions. Such sensors have been designed for several metabolites and second messengers, including cytosolic calcium, and more recently, in Verkhusha's hands they have been applied to detect intracellular $\mathrm{pH}$ changes and phosphatidylinositol 3-phosphate.

Evrogen also offers a calcium sensor called Case12, which is not based on

\section{BOX 2 COLORFUL INTERACTIONS}

By its nature as a phenomenon that manifests when two components are in close proximity, FRET is an interesting tool to detect protein-protein interactions in a cell. The two FRET partners can be fused to two protein interaction partners so that they will generate a FRET signal only when the two proteins of interest are associated. But although the theory of FRET sounds clean, simple and straightforward, it does not always work this way in practice. "The most fundamental problem is we want to use light microscopy because this is compatible with living cells," says Kees Jalink, group leader in cell biology at the Netherlands Cancer Institute, "however, light microscopy has about $1 / 4$ of a micrometer resolution, which is about two orders of magnitude above the molecular resolution." Scientists use FRET to boost the 'resolution' of optical microscopy to about $5 \mathrm{~nm}$, leading FRET to be known as the molecular ruler. "But in fact this is not true and very confusing," explains Jalink. "The amount of FRET depends not just on the inverse sixth power of distance, because in practice it is also strongly dependent on the orientation and on other factors like ionic strength or the presence of facilitators." Other frustrating artifacts of FRET such as bleaching, phototoxicity, instrumental and algorithm imperfections, imperfections in the Forster FRET theory, collisional FRET, also called spurious or crowding FRET, have also been noted, making it a very peculiar technique that can be exact but certainly not simple.

Other grumblings about FRET were heard from Bob Bulleit, director of research and development at Promega. "FRET is a very temperamental technology," says Bulleit. "And it's not very efficient, particularly when you get into the context and the complexity of all the proteins in the environment." Bulleit predicts that people will find a way around this challenge by using protein complementation assays. In these assays, two fragments of a reporter protein are fused to each of two protein partners of interest. As the proteins interact in the cell, the two halves of the reporter are brought together and reconstitute the marker function, which can be enzymatic activity, fluorescence or bioluminescence.

Several complementation pairs, providing different readouts, have been developed in academic labs. On the commercial front, MBL International has just released a new kit, the CoralHue Fluo-Chase kit, for detecting proteinprotein interactions using protein complementation of their new monomeric reporter protein, Kusabira Green (mKG). 
FRET. Rather, Case12's name derives from the fact that calcium binding causes its brightness to increase by up to 12 -fold. According to Evrogen, Case 12 can be used to generate stable cell lines and transgenic animals.

\section{Pushing the limits}

Although FRET is still difficult to perform

in high-throughput settings, fluorescence is by nature a very practical readout for high-throughput assays, and fluorescent proteins are increasingly popular with the growing interest in high-throughput screening, particularly in drug discovery applications. Suvrana Gandlur, product manager for fluorescent proteins at Clontech, believes that luminescent tags will also have a role in these applications. "The development of multi-detection instruments like the SpectraMax M5 from Molecular Devices makes it possible to detect and measure multiple events like fluorescence and chemiluminescence nearly simultaneously," says Gandlur who envisions such combinations as powerful readouts for live cell imaging, such as the study of translocation and secretion.

To assist with high-throughput assays, Clontech now offers cell lines stably transfected with different biosensors. The ZsGreen Proteasome Sensor and HT-1080 Retro DsRed-Express (invasion assay) cell lines offer consistent expression, which helps simplify data interpretation, says Gandlur. The proteasome sensor allows the researcher to monitor cellular proteasome activity by measuring the green fluorescence intensity of the sensor in the cell using methods such as flow cytometry analysis. It allows monitoring in a noninvasive, 'on the fly' manner using live cells. The red fluorescent cells of the HT-1080 Retro dsRed-express cell lines are easily visualized after migration through a membrane by simple fluorescence microscopy.

\section{A bright future}

It is exciting to see new developments inching the field of fluorescent proteins forward each year, but will this be accompanied by the occasional leap? "There is no question that use of fluorescent proteins as passive labels has led to a complete revolution in cell biology," says Remington. "However, the use of fluorescent-protein biosensors and photoactivatable fluorescent proteins is still at such an early stage that it is difficult to point to any experiments that have resulted in a genuine breakthrough in our understanding of cell biology or to a radical change in our thinking. But I have no doubt that this will happen."

1. Eisenstein, M. Nat. Methods 3, 647-655 (2006).

2. Mena, M.A. et al. Nat. Biotechnol. 24, 1569-1571 (2006).

3. Ai, H.W. et al. Biochem. 46, 5904-5910 (2007).

Caitlin Smith is a science writer based in Portland, Oregon (caitlin.smith@comcast. net). 


\section{SUPPLIERS GUIDE: COMPANIES OFFERING TOOLS FOR FLUORESCENCE IMAGING}

\section{Company}

Active Motif

AnaSpec

Andor Technology

Applied Precision

BD Pharmingen

Biocat

\section{BioPAL}

BioVision

Cambridge Research \& Instrumentation (CRi)

Carl Zeiss

Chroma Technology

Clontech

Covalys

Evident Technologies

Evrogen

GE Healthcare

Hamamatsu

HORIBA Jobin Yvon

Intracellular Imaging

Invitrogen/Molecular Probes

ISS

Kodak

Leica Microsystems

LI-COR Biosciences

Lightools

LUX biotechnology

MAG Biosystems

MBL International

MCID Scientific Imaging Software

Media Cybernetics

Molecular Devices

Nanoco Technologies

Nikon

Olympus America

Omega Optical

PerkinElmer

Pierce Biotechnology

Prior Scientific

Promega

Roche Applied Sciences

Semrock

Stratagene

UVP

VisEn Medical

Visiopharm

\section{Web address}

http://www.activemotif.com

http://www.anaspec.com

http://www.andor.com/

http://www.api.com

http://www.bdbiosciences.com

http://www.biocat.de/

http://www.biopal.com/

http://www.biovision.com

http://www.cri-inc.com/

http://www.zeiss.com/

http://www.chroma.com

http://www.clontech.com/

http://www.covalys.com/

http://www.evidenttech.com/

http://www.evrogen.com/

http://www.amershambiosciences.com,

http://www.hamamatsu.com,

http://www.jobinyvon.com

http://www.intracellular.com,

http://www.invitrogen.com,

http://www.iss.com

http://www.kodak.com/

http://www.leica-microsystems.com/

http://www.licor.com

http://www.lightools.com/

http://www.luxbiotech.com,

http://www.magbiosystems.com/

http://www.mblintl.com/

http://www.mcid.co.ul

http://www.mediacy.com

http://www.moleculardevices.com/

http://www.nanocotechnologies.com

http://www.nikon.com,

http://www.olympusamerica.com/

https://www.omegafilters.com/

http://www.perkinelmer.com,

http://www.piercenet.com/

http://www.prior.com

http://www.promega.com/

http://www.roche-applied-sciences.com

http://www.semrock.com/

http://www.stratagene.coms

http://www.uvp.com/

http://www.visenmedical.com/

http://www.visiopharm.com 